\title{
Erlauntza: calidad de vida en el barrio a través del impulso a la confianza entre profesionales
}

\section{Adriana Martínez Sans}

Asociación para la Promoción de la Tecnología Social (Aptes)

adriana@tecnologiasocial.org

\section{Aitziber Etxezarreta}

Departamento de Salud y Medio Ambiente, Ayuntamiento de Donostia-San Sebastián

\section{Sorkunde Jaca}

Departamento de Salud y Medio Ambiente, Ayuntamiento de Donostia-San Sebastián

\begin{abstract}
Artikulu honetan Erlauntza proiektuaren berri ematen da, Donostia Lagunkoia/Biztanle Adinarekin Lagunkoia den Hiri Planaren esparruan sortutakoa, Donostiako Udalak hiri-bizimoduaren arlo guztietan biztanleriaren zahartzeak ekarriko dituen erronkak zeintzuk izan daitezkeen planteatzen du. Planean urte batzuetan egindako lan handiaren ondoren, ezinbestekotzat hartu zen auzoetan pertsona adinduei zerbitzuak eskaintzen zizkieten profesionalei konfiantzan lan egitea eta ongizateikuspegi komunitarioaren hastapenak izatea. Erlauntza auzoetan jarduten duen profesionaltalde baten izena da, bi hilez behin elkartzen dena, eta haien asmo bateratua pertsonen bizikalitatea sustatu, mantendu edo berreskuratzea $\mathrm{da}$, bakoitzari dagokion jardueraren bitartez, eta zerbitzu-helburuak hobe lortzea ahalbidetzen dien elkarrekiko konfiantza handiko erlazioa sortarazita.
\end{abstract}

\section{GAKO-HITZAK:}

Bizi-kalitatea, profesionalak, konfiantza, osasunerako aktiboak, osasunerako toki-sareak, metodologia
Este artículo presenta el proyecto Erlauntza, surgido en el marco de Donostia Lagunkoia/Plan de Ciudad Amigable con la Edad, una iniciativa del Ayuntamiento de Donostia-San Sebastián que pone el foco en los retos que plantea el envejecimiento de la población en todos los ámbitos de la vida urbana. Después de unos años de trabajo intenso en el plan, se consideró indispensable que las y los profesionales que ofrecían servicios - públicos y privados- a las personas mayores en los barrios trabajaran en clave de confianza y se iniciaran en la perspectiva comunitaria del bienestar. Erlauntza - “colmena" en euskera- es la denominación del grupo de personas profesionales con actividad en los barrios que, reunidas de manera bimestral, comparten el propósito de fomentar, mantener o recuperar la calidad de vida de las personas a través de su actividad respectiva y crean una relación de alta confianza mutua entre ellas que les permite lograr mejor sus objetivos de servicio.

\section{Palabras Clave:}

Calidad de vida, profesionales, confianza, activos para la salud, redes locales para la salud, metodología. 


\section{Las redes locales de salud, una herramienta útil para el abordaje comunitario de la salud}

Tal como recoge con detalle la Guía metodológica para el abordaje de la salud desde una perspectiva comunitaria (Departamento de Salud del Gobierno Vasco, 2010), salud comunitaria es la expresión colectiva de la salud de los individuos y grupos en una comunidad definida, determinada por la interacción entre las características de los individuos, las familias y el medio social, cultural y ambiental, así como por los servicios de salud y la influencia de factores sociales, políticos y globales.

Dicha guía es un marco teórico de actuación que facilita incorporar la perspectiva comunitaria a las instituciones de salud implicadas en la promoción de la salud, la orientación hacia los determinantes de la salud, a la perspectiva de equidad y participación (Departamento de Salud del Gobierno Vasco, 2013) y al trabajo desde una metodología de activos para la salud (Argüelles et al., 2016). Un nuevo enfoque de salud más amplio, basado en los aspectos positivos de individuos y comunidades, que se centra en identificar aquello que sí funciona y que hace fuerte al individuo o grupo, y potenciarlo. Ayuda también a identificar aquello que la comunidad no identifica como activo, y permite investigar los motivos por los que estos recursos no son valorados como positivos por la comunidad, así como iniciar un proceso a través del cual se reconviertan en activos o se pueda reorientar los recursos hacia nuevas necesidades (Hernán et al., 2010). La perspectiva de salud positiva es un enfoque basado en activos y recursos de las comunidades, en lugar de en necesidades y deficiencias.

Con base en ello, la guía propone implementar acciones que puedan mejorar el bienestar aprovechando los recursos existentes. Esto se concreta abordando un trabajo en red a nivel local que consiste en crear alianzas, sinergias entre diferentes agentes, para establecer objetivos comunes y actuar cooperativamente para alcanzarlos, de forma que los recursos sean mejor aprovechados.

Estas redes locales para la salud agrupan a los diferentes agentes de la comunidad (Administración, recursos profesionales y técnicos y ciudadanía) organizados de forma no jerárquica en torno a temas o preocupaciones comunes, que tienen una incidencia directa en la salud de la comunidad y que se abordan de manera activa y sistemática, evitando duplicidades y fomentando el trabajo conjunto sobre la base del compromiso y la confianza. Surge así un contexto muy apropiado en el que identificar, comprender y ejercitar los elementos que facilitan el compromiso y la confianza necesarios para que este trabajo conjunto sea posible.

\section{2. ¿Qué es Erlauntza?}

Son muchas las personas profesionales que desde su día a día orientan, informan o cuidan a quienes acuden a los servicios que se ofrecen en los barrios, desde contextos o en sectores diferentes: centro de salud, polideportivo, centro de servicios sociales, actividades de envejecimiento activo, casa de cultura, farmacias, etc. Todas estas profesionales comparten el propósito de fomentar, mantener 0 recuperar la calidad de vida de las personas mayores.

Con frecuencia ocurre que no se dispone de tiempo para que las personas que están alrededor de la ciudadanía ofertando servicios se conozcan entre ellas, qué actividades se programan, o las que se están pensando en incorporar. Esto hace que la ciudadanía de todas las edades, pero especialmente las personas mayores del barrio, no puedan aprovechar todo lo que tienen a su alrededor para aumentar o mantener su calidad de vida. Si bien existen foros de coordinación a nivel "meso" (responsables de servicio a nivel territorial, comarcal o municipal), se observa que el nivel "micro" (profesionales a pie de persona y servicio en los barrios), no dispone de ningún foro de encuentro y es clave para avanzar en intervenciones intersectoriales locales. Es ahí donde aparece la oportunidad de construir una relación profesional basada en el compromiso y la confianza y que el equipo promotor de esta iniciativa venimos impulsando desde 2017.

Erlauntza es una iniciativa originalmente promovida por la Asociación para la Promoción de la Tecnología Social (Aptes), enmarcada en el proyecto Euskaltrust, que ha contado para su germinación con el apoyo de la Diputación Foral de Gipuzkoa a través del programa Etorkizuna Eraikiz. Euskaltrust tiene por objetivo hacer de la confianza un valor social reconocido, a nivel personal, grupal e institucional. Desde Euskaltrust se propone la iniciativa Erlauntza como ámbito de trabajo concreto en relación con la construcción de relaciones de confianza entre personas y organizaciones.

El objetivo general de Erlauntza es activar relaciones de alta confianza mutua entre las personas que trabajan en los barrios desde distintos servicios a las personas, fundamentalmente mayores, pero no de manera exclusiva. Esto se concreta en iniciar la construcción de grupos Erlauntza en los barrios de Donostia, compuestos por responsables de proximidad de los distintos servicios sanitarios, sociales, culturales, deportivos y de envejecimiento activo, y lograr entre estas personas altos niveles de confianza que permitan a cada una desde su espacio (físico) y ámbito (función) lograr mejor sus objetivos de servicio.

Erlauntza está facilitando el encuentro de grupos locales de profesionales que se conocen, se reconocen en su especialidad y se relacionan de manera sostenida para reforzar el servicio a las personas mayores del barrio a través del papel de cada profesional y de la actividad de la entidad a la que representa. Erlauntza es un trabajo artesanal, en la medida en la que es preciso invertir tiempo en adquirir un mayor conocimiento de las personas, 
de los programas organizados, de los que están en previsión de organizarse y de los recursos tangibles e intangibles disponibles.

Los objetivos específicos de Erlauntza son los siguientes:

- Aumentar el conocimiento mutuo personal e institucional.

- Conocer instalaciones y personas de los diversos recursos del barrio.

- Crear conexiones que generan ventajas para el trabajo que desarrolla cada profesional.

- Consolidar esas conexiones en forma de prácticas cíclicas (procesos) que amplifiquen los resultados.

- Reconocer los buenos casos en público.

- Crear herramientas (analógicas o digitales) que consoliden las relaciones.

- Elevar resultados a niveles superiores en cada servicio de proximidad.

En los siguientes epígrafes se describe la evolución del proyecto a lo largo de tres fases, así como las metodologías utilizadas en ellas para impulsar el trabajo en red y evaluar sus resultados. El artículo termina con una serie de conclusiones.

\section{Primera fase: grupo promotor}

La iniciativa Erlauntza abordó su primera fase desde junio hasta diciembre de 2017. Consistió en la creación de un grupo promotor constituido por:

- Representantes técnicos del Colegio Oficial de Farmacia de Gipuzkoa: Estíbaliz Goyenechea y Amaia Malet.

- Responsable de coordinación sociosanitaria de la organización sanitaria integrada (OSI) Donostialdea: Mari Jose Goñi.

- Responsable de Centros de Servicios Sociales del Ayuntamiento de San Sebastián: Marijo Larrea.

- Coordinadora del Plan Donostia Lagunkoia en Ayuntamiento de San Sebastián: Eva Salaberria.

- Responsable de Actividades Deportivas del Patronato Municipal de Deportes de Donostia: Iñaki Zabaleta.

- Responsable de Servicio de Participación Activa y Tiempo Libre (Plus 55), María Fernández.

- Técnicas del Promoción de la Salud, Subdirección de Salud Pública, Departamento de Salud del Gobierno Vasco: Arantxa Uranga y María Blanco.

- Profesional independiente: Anjel Irastorza.

- Equipo de Aptes: Juanjo Goñi y Adriana Martínez.

Este grupo promotor trabajó en tres sesiones para conocerse y alinear el propósito compartido, de manera que nos permitiera más adelante empezar a trabajar en forma local de barrio.

En la primera sesión (12 junio 2017), se trabajó en el conocimiento mutuo de las personas involucradas y se inició la caracterización de una organización de implicación recíproca, esto es, la que tiene la capacidad para influir en el futuro de la otra: cuando haya que optar por varias posibilidades, cuando haya que diseñar, cuando haya que evaluar, etc. Asimismo, se iniciaba el esbozo del propósito que une a las personas participantes, algo que se trasladó a la siguiente sesión.

En la segunda sesión (26 julio 2017), se estableció el propósito compartido de este grupo promotor definido finalmente como "Fomento, mantenimiento o recuperación de la calidad de vida de las personas mayores, desde una óptica pluridisciplinar, pluriinstitucional y con foco en la persona". Lógicamente, aparece la importancia de que este propósito común se concrete con la acción de personas con perfiles más operativos, que son los que están en contacto con las personas a las que se dirigen los distintos servicios. Este encuentro sirvió también para reconocer que el incremento de la confianza entre agentes fomenta la identificación y ejecución práctica de mejoras de los procesos de atención a personas, algo que ocurre en iniciativas ya en marcha, pero aún insuficientes. Intensificar estas relaciones de confianza supone ventajas sobre las situaciones actuales.

Un aspecto importante trabajado con este grupo promotor ha sido lo relativo a los distintos niveles de vinculación que desde la iniciativa de la Escuela de Diseño Social de Aptes ${ }^{1}$ consideramos de interés para generar un entendimiento mínimo. Si queremos ser especialistas en la cooperación, necesitamos distinguir la participación de la colaboración, de la cooperación y conocer que la implicación recíproca ${ }^{2}$ es el nivel máximo de vinculación entre personas y entidades. Por último, y respecto a los ámbitos de trabajo y las formas de abordarlos, el grupo promotor identificaba un buen número de ellos, que agrupamos en tres:

- Responder a la necesidad de saber unas organizaciones de otras en sus iniciativas y oportunidades de disponer de recursos entre organizaciones. Recursos limitados y demandas crecientes.

- Mejorar los mecanismos de buena orientación a la persona destinataria del servicio, evitando sobrecargas y el peloteo de un lado a otro.

- Señalar la intervención precoz en la proximidad como foco de generación de ventajas y reducción de costes venideros. 
La tercera sesión (4 octubre 2017) se dedicó a trabajar de manera individual la Balanza de Valor ${ }^{3}$ de Erlauntza: el valor neto (lo que recibe menos lo que pone) que supone esta iniciativa para cada persona y la entidad a la que representa en este grupo promotor: “¿Qué quieres que Erlauntza aporte para el desarrollo de tu servicio? ¿Qué tienes que aportar tú y tu servicio a Erlauntza?”.

Del uso de la herramienta de la Balanza del Valor Social de Erlauntza durante esta sesión de trabajo surgieron los ámbitos concretos en los que un grupo Erlauntza de barrio debería poner el foco:

1. Operativo: encuentros programados de cada grupo Erlauntza. En ellos, cada entidad comparte cuáles son los asuntos que le ocupan en la próxima quincena, proyectos que pone en marcha, campañas que va a desplegar, problemas surgidos, etc. El objetivo es que todas las personas del Erlauntza estén informadas de lo operativo: qué ocupa o va a ocupar a las otras en las próximas semanas. De ahí surgirán peticiones y acciones de apoyo o propuestas de mejora en dichas acciones. Comprende acciones de coordinación, sincronización y mejoras en las prácticas vigentes. Es importante diseñar el encuentro para que no se alargue en exceso (un máximo de dos horas). Es un encuentro abierto a las personas que, formando parte del grupo promotor y no estando en primera línea de actividad, puedan acudir a él para aproximarse al terreno.

2. Apoyo en recursos: en esta línea se pueden considerar aportaciones de recursos de información, instalaciones, material de programas, breves asesoramientos que pudieran intercambiarse entre los miembros del Erlauntza, etc. Es beneficioso que se opte por reuniones rotatorias en los centros de trabajo de los participantes y se aproveche para conocer instalaciones, equipamientos y reformas que se emprenden. Es también la ocasión para presentar a las personas del Erlauntza a personal del centro visitado, como miembros técnicos o directivos.

3. Más allá: en esta línea se puede tratar las colaboraciones en la elaboración y diseño de planes de acción. Puede comprender el diseño de acciones conjuntas y la colaboración en programas de trabajo interinstitucional. Este es un espacio de trabajo con miembros del grupo promotor y de formalización de buenas prácticas en procesos estables. Es la ocasión para compartir experiencias entre grupos Erlauntza y evaluar la marcha del proyecto y sus resultados.

4. Aprendizaje de las personas de los grupos Erlauntza: esta línea de acción para su empoderamiento debe comprender aportaciones en apoyo formativo en métodos de trabajo, uso

${ }^{3}$ http://www.escueladisenosocial.org/soportes-formativos/ la-balanza-de-valor/. de herramientas y de casos de éxito y situaciones de carencias que deban ser analizadas y resueltas a través de cambios en disponibilidad de información, mejores coordinaciones o sistemas de información más eficientes. Esta línea de trabajo debe conectarse con los planes de formación y mejora de los procesos de las diferentes entidades partícipes, siendo muy valiosa la participación de personas de distintas entidades del Erlauntza en talleres de resolución de casos.

Con este recorrido realizado en 2017 con -recordamos- las personas con responsabilidades de dirección o coordinación de los distintos servicios, planteamos la fase 2, de acción en lo local. Para ello, se identificaron en el grupo promotor los barrios de Egia e Intxaurrondo como los lugares en los que iniciar la construcción de grupos Erlauntza.

\section{Segunda fase: grupos locales Erlauntza Egia y Erlauntza Intxaurrondo}

A partir de enero de 2018 y hasta diciembre de $\mathbf{2 0 1 8 ,}$ con el apoyo continuado de Donostia Lagunkoia y en coordinación con las personas del grupo promotor, se identificó y convocó a las profesionales de los distintos ámbitos implicados en la atención a las personas mayores de Egia e Intxaurrondo, respectivamente. La convocatoria y celebración de las sesiones Erlauntza en Egia e Intxaurrondo durante esta segunda fase se realiza y facilita desde Aptes, contando con financiación para esta dedicación de la Diputación Foral de Gipuzkoa del proyecto Euskaltrust en el marco de Etorkizuna Eraikiz.

La primera reunión Erlauntza, cebrada en cada barrio (en Egia el 31/1/18 y en Intxaurrondo el $7 / 2 / 18$ ), consistía en conocer y poner cara a todos los participantes, saber en qué y dónde trabajan, qué inquietudes tienen, de manera que se inicie la relación (o reinicie, en el caso de que ya existiera) entre los profesionales de distintos ámbitos del barrio. Se organizaba una carpeta de trabajo compartido en el servicio Google Drive, con los distintos documentos generados y, en especial, el listado con las coordenadas de las personas participantes.

A partir de la segunda reunión Erlauntza en ambos barrios se estableció un orden del día estandarizado para todas las sesiones:

1. Relaciones: Contactos habidos en el periodo anterior: resultados, fallos y mejora de las conexiones.

2. Planes: para las próximas 8 semanas. Peticiones de apoyo mutuo. Informaciones de interés para las personas Erlauntza.

3. Cosas nuevas: generación de iniciativas entre personas asistentes. Nuevas ideas. Proyectos piloto. 
4. Reconocimiento: Buenas prácticas que funcionan. Herramientas de coordinación.

5. Recursos y personas: visita a instalaciones y presentación de personas del centro que acoge.

La periodicidad y duración de las sesiones - bimestral y dos horas - fue acordada por los grupos y se logró una buena acogida a la propuesta de celebrarlas de manera rotatoria en diferentes lugares del barrio.

Los perfiles de las personas que están participando en Erlauntza Egia y Erlauntza Intxaurrondo son: trabajadora social del Centro de Servicios Sociales, enfermera de enlace sociosanitario del Centro de Salud, técnico de proximidad de Donostia Kirola, técnicas de Promoción de la Salud del Ayuntamiento de Donostia, técnicas del programa Plus55, titulares de farmacias Lagunkoia del barrio, técnicas del Departamento de Salud Pública del Gobierno Vasco y responsables de la Casa de Cultura (Donostia Kultura). Como se puede ver, todas ellas personas de perfil técnico y con contacto directo con las personas en el barrio a través de sus servicios.

Con la experiencia de un año de trabajo de los grupos Erlauntza de Egia e Intxaurrondo, se logró ya durante 2018 un importante número de colaboraciones cruzadas entre participantes, acciones conjuntas, retos para los que se diseña una respuesta coordinada, incremento en el conocimiento de las distintas realidades sociales del barrio, etc. Y, por supuesto, un incremento en calidad y cantidad de la relación entre las profesionales y, por tanto, de la confianza mutua entre ellas. En el Cuadro 1 se enumeran algunas de ellas.

Estos ejemplos son solo una muestra de un constante intercambio de información, solicitud de apoyos entre servicios y diseños y desarrollos de planes conjuntos.
Un aprendizaje importante se deriva de cómo los grupos locales generan relaciones entre ellos. Por ejemplo, las farmacéuticas involucradas en los Erlauntza de Egia e Intxaurrondo preparan y comparten los contenidos de las charlas en un repositorio que se pone a disposición a otras profesionales colegiadas en el Colegio Oficial de Farmacia de Gipuzkoa, para poder disponer de estos en el momento de extenderlas a otros barrios. Otro aprendizaje surge de las cuestiones que se traen a los grupos Erlauntza por parte de las profesionales y que las superan en su capacidad de acción y son derivadas a instancias superiores de las organizaciones implicadas. Por ejemplo, en Erlauntza Gros se ha acordado entre los profesionales del Centro de Salud y las farmacias la incorporación de una alerta en el aplicativo de los primeros para conocer a aquellos pacientes que utilizan pastilleros preparados por las farmacias (SPD) y para los que un cambio de medicación supone deshacer los pastilleros ya preparados en las farmacias con antelación.

\section{Tercera fase: relevo de liderazgo, despliegue de grupos locales, medición de avance}

Desde junio de 2018 y hasta la fecha, el avance de Erlauntza ha sido muy relevante en tanto que se han producido dos hitos clave: el relevo en su liderazgo y su despliegue a más barrios de la ciudad.

Aptes proponía en junio de 2018 al Departamento de Salud y Medio Ambiente del Ayuntamiento de Donostia-San Sebastián que el equipo de profesionales dedicadas a la Promoción de la Salud dentro de él y participantes desde un inicio en los dos grupos Erlauntza de barrio tomaran el testigo de consolidar estos dos grupos ya iniciados y comenzar de manera paulatina el despliegue de esta iniciativa en otros barrios de la ciudad. Esto se validó con

Cuadro 1. Algunas colaboraciones llevadas a cabo por los grupos Erlauntza de Egia e Intxaurrondo (Donostia-San Sebastián)

\begin{tabular}{|l|l|}
\hline Colaboraciones & Ejemplo \\
\hline Derivaciones de personas a diferentes servicios & $\begin{array}{l}\text { Desde la farmacia se indica a un cliente la oportunidad de solicitar la participación } \\
\text { en un programa de actividad física dirigida -Piztu, de Donostia Kirola- a través de } \\
\text { la prescripción médica. }\end{array}$ \\
\hline Diseño de programa de charlas y su impartición & $\begin{array}{l}\text { Charlas sobre incontinencia organizadas por el Servicio de Participación Activa y } \\
\text { Tiempo Libre (Plus 55) en colaboración con asociaciones de mayores e impartida } \\
\text { por farmacéuticas del barrio. }\end{array}$ \\
\hline $\begin{array}{l}\text { Diseño de prueba piloto del servicio de preparación } \\
\text { de medicación en blisters (SPD) a colectivos } \\
\text { susceptibles de mejorar su adhesión al tratamiento } \\
\text { mediante esta propuesta }\end{array}$ & En estado de valoración una prueba piloto en Egia y Gros. \\
\hline $\begin{array}{l}\text { Comunicación entre los distintos profesionales de las } \\
\text { actividades programadas para cada mes }\end{array}$ & $\begin{array}{l}\text { El Servicio de Participación Activa y Tiempo Libre (Plus 55) envía folletos de la } \\
\text { actividad a cada farmacia. }\end{array}$ \\
\hline $\begin{array}{l}\text { Comunicación sobre perfiles presentes en el barrio } \\
\text { que no toman parte en las actividades deportivas, } \\
\text { culturales o de ocio. }\end{array}$ & $\begin{array}{l}\text { La Casa de Cultura incorpora la mirada a las personas mayores que se descuelgan } \\
\text { de la oferta cultural, a pesar de que anteriormente sí habían disfrutado de ella. }\end{array}$ \\
\hline $\begin{array}{l}\text { Impulso en el despliegue de iniciativas promovidas } \\
\text { por distintos servicios. }\end{array}$ & $\begin{array}{l}\text { Despliegue de Piztu, de Donostia Kirola, en el barrio de Egia con profesionales en el } \\
\text { mismo Centro de Atención Primaria de la OSI Donostialdea. }\end{array}$ \\
\hline
\end{tabular}

Fuente: Elaboración propia. 
ocasión de la reunión en el mes de junio de 2018 del grupo motor de la iniciativa, aquel que en 2017 había desarrollado el diseño inicial. El compromiso de Aptes desde el mes de septiembre de 2018 ha sido acompañar al equipo de Promoción de la Salud en esta nueva etapa, pero sin desempeñar el rol de líder.

El despliegue en más barrios fue iniciado en septiembre de 2018 , de nuevo con un importante trabajo de identificación y convocatoria a profesionales, en este caso, de los barrios de Parte Vieja y Gros, escogidos por el grupo motor en base a criterios como la necesidad y disponibilidad de red de profesionales sensibles a una iniciativa como Erlauntza. Así, después de la primera reunión de presentación en ambos barrios, durante los meses de diciembre de 2018 y enero de 2019 se han celebrado las reuniones con el orden del día previsto de manera estándar.

Finalmente, esta tercera etapa ha permitido generar una herramienta de medición del avance de la iniciativa habida cuenta que ya había un recorrido realizado por dos grupos. Este tipo de iniciativas que buscan mejorar en la calidad de las relaciones entre personas son complejas, de personas y entidades que no son estáticas, y tampoco tienen explicitado dónde va a llegar. Por ello, se requiere de una herramienta ad hoc y cualitativa, que se ha denominado Erlauntzometro ${ }^{4}$.
Así, se ha trabajado en identificar entre las personas involucradas en los grupos Erlauntza con mayor recorrido las doce dimensiones que agrupadas pretenden medir, tanto el clima interno del grupo, como la sistemática y procesos, la gestión de recursos y los resultados e impacto generado.

Cada dimensión cuenta con una descripción de cuatro niveles de práctica: dos son malos o no aportan nada a hacer crecer esa dimensión y dos son buenos y/o sí aportan a ella. Sobre una hoja de cálculo preparada a tal efecto reflejamos la frecuencia con la que se da cada nivel de práctica: o indica ausencia total de dicha situación o comportamiento; 100 indica que esta situación o comportamiento está totalmente extendido y aplicado. Los valores intermedios representan la proporción de situaciones o personas que cumplen dicha afirmación: muy pocas, 20; la mitad de los casos, 50; la mayoría, 80; etc. Tras aplicar este instrumento se obtiene una representación de la evaluación como la que se muestra en el Gráfico 1:

En enero de 2019 se ha iniciado la medición del avance en los grupos Erlauntza de Egia e Intxaurrondo, respectivamente, con más recorrido que los incorporados posteriormente. Esta medición se hace de manera paulatina, esto es, en cada sesión Erlauntza se aborda una única categoría, de manera que no se detrae excesivo tiempo de las reuniones

Gráfico 1. Representación visual de los resultados del Erlauntzometro*

Consolidación de las relaciones

de transparencia y confianza

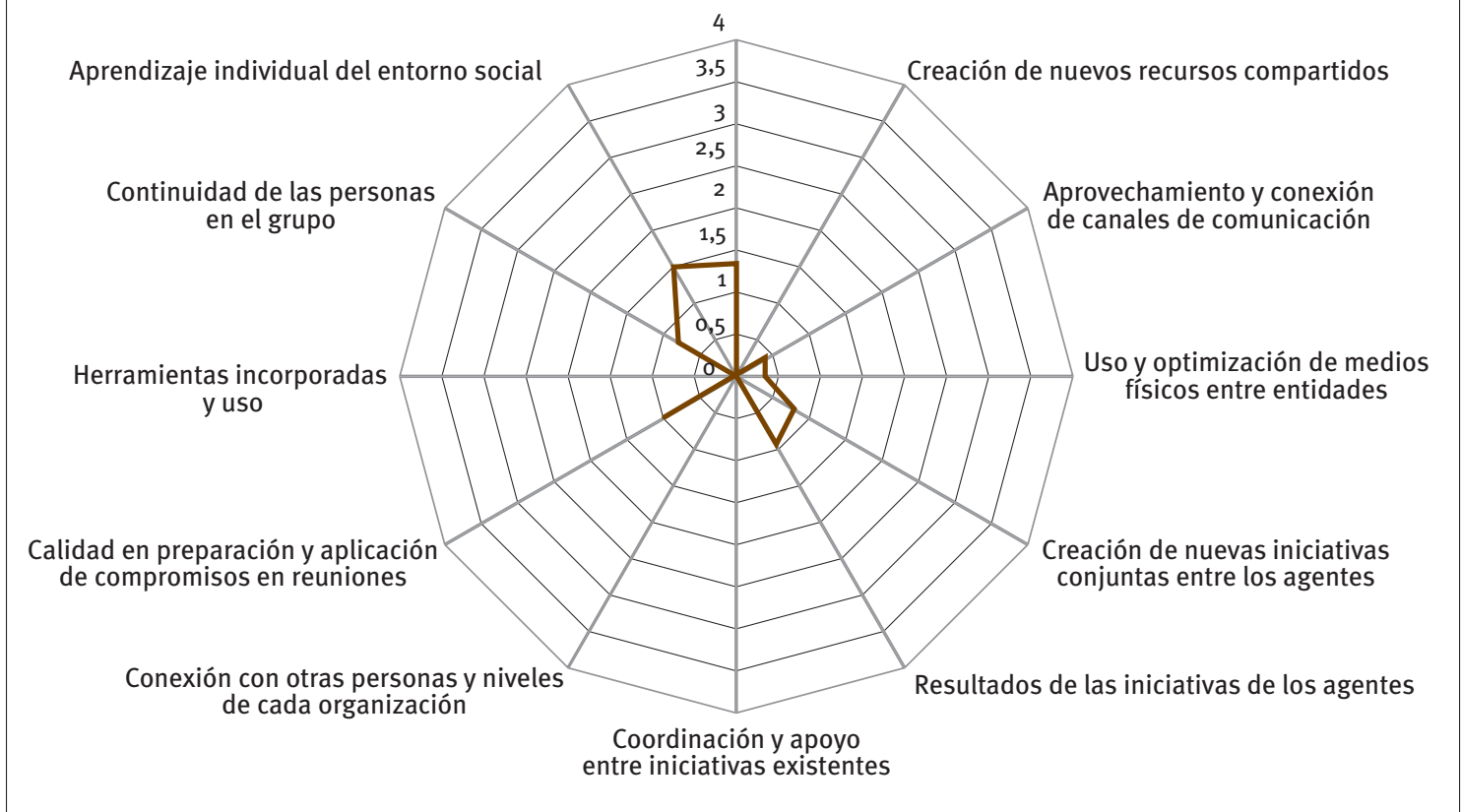

* Datos ficticios, utilizados a título meramente ilustrativo.

Fuente: Elaboración propia.

4 http://www.escueladisenosocial.org/blog/2019/05/08/ diseno-de-una-metrica-de-avance-adhoc-para-erlauntza/. 
corrientes. Es importante señalar que el valor del Erlauntzometro está especialmente en que permite ordenar la reflexión conjunta del grupo, recogiéndose no solo la puntuación numérica de la frecuencia de esa actitud, sino también los matices y valoraciones que hacen todas las personas.

\section{Conclusiones}

La hipótesis de partida de Euskaltrust en general, y de su aplicación a Erlauntza en particular, se ha confirmado: sí es posible obtener mejoras sustanciales en las actividades operativas, si se activan mecanismos de fomento de la confianza, es decir, compartiendo información, aumentando el conocimiento interpersonal y concretando un propósito común. No obstante, esto solo es posible si se invierte tiempo y método en la iniciativa: no hay aumento de confianza si no ha habido inversión de recursos, y esto solo es posible con el apoyo de los equipos directivos intermedios de las organizaciones a las que pertenecen las personas implicadas en Erlauntza, para lograr estas relaciones de alta confianza mutua, que además alienten su práctica de manera habitual en sus procesos. Esta andadura ha permitido identificar seis ejes de conclusiones finales, que se desarrollan a continuación:

1. Eje eficacia/coste: es posible obtener mejoras sustanciales en las actividades operativas si se activan mecanismos de fomento de la confianza, es decir, compartiendo información, aumentando el conocimiento interpersonal y concretando un propósito común tanto en las relaciones internas como externas de las organizaciones.

2. Eje método y sistemática: lo anterior es posible y genera resultados si se le dedica tiempo y método específico a Erlauntza. El método requiere del empleo de herramientas compartidas. Las pautas básicas son: conocimiento mutuo, declaración de intereses, comunicación sistemática, compartición de recursos y diseño compartido de futuras acciones.
3. Eje apoyo directivo: no es posible avanzar con resultados en esta cooperación horizontal sin el apoyo de los equipos directivos intermedios de las organizaciones a las que pertenecen las profesionales implicadas. Este apoyo es sobre todo en tiempo de dedicación, que se verá compensado en mayor eficacia en el logro de los objetivos individuales, con un lógico decalaje en el tiempo.

4. Eje valor de este trabajo: la activación de estas relaciones es posible tanto entre organizaciones diversas como en el interior de una organización. Sus resultados dependerán de cómo se consolide como una actividad más, que sea normal en la agenda de trabajo de las personas. Esta relación estable y las dinámicas que generan son fuentes permanentes de innovaciones operativas de aplicación muy directa en los objetivos de las entidades.

\section{Eje especialistas de apoyo y cualificación} profesional: estas experiencias apuntan a que la creación de funciones intraorganización o extraorganización con la misión de crear y desarrollar vínculos de confianza en el plano operativo entre agentes diversos que tienen un foco de acción común, como puede ser un oficio, con el bagaje de las herramientas de la Escuela de Diseño Social que se han ensayado con éxito en los casos citados.

6. Eje inserción de métricas: la inserción de estas prácticas de trabajo en los modelos de gestión requiere introducir objetivos e indicadores, pero a su debido tiempo. En este sentido, en esta etapa de germinación, se ha podido hacer un diseño de métrica ad hoc en contextos complejos para el caso de Erlauntza. A partir de las técnicas propuestas en la Escuela de Diseño Social, en esta etapa de germinación se han incorporado alguna métrica específica de contextos complejos. Concretamente se ha creado el Erlauntzometro. 


\begin{tabular}{|c|c|c|}
\hline & ¿Qué necesito yo/mi organización de Erlauntza? & ¿Qué puedo aportar yo/mi organización a Erlauntza? \\
\hline \multirow{3}{*}{$\begin{array}{l}\text { Departamento } \\
\text { de Salud } \\
\text { Pública }\end{array}$} & $\begin{array}{l}\text { Problemas: } \\
\text { - Limitado número de personal técnico para trabajar en el } \\
\text { terreno. } \\
\text { - Ubicarlo en el marco de las líneas concretas del } \\
\text { Departamento. }\end{array}$ & \multirow{3}{*}{$\begin{array}{l}\text { - Conocimientos. } \\
\text { - Experiencias de éxito en otros municipios. } \\
\text { - Olusión. } \\
\text { aydenes de ayudas a iniciativas de este tipo para los } \\
\text { ayuntantos. }\end{array}$} \\
\hline & $\begin{array}{l}\text { Necesidades: } \\
\text { - Conocer los proyectos de otros departamentos. } \\
\text { - Mejorar la coordinación con ellos. } \\
\text { - Contar con una interlocución concreta en el Ayuntamiento } \\
\text { de Donostia-San Sebastián. } \\
\text { - Definir el proyecto de manera concreta. } \\
\text { - Diferenciar entre el proyecto en lo local y las relaciones a } \\
\text { nivel intermedio-directivo. } \\
\text { - Evaluación. }\end{array}$ & \\
\hline & $\begin{array}{l}\text { Planes: } \\
\text { - Desarrollar el abordaje de salud comunitaria. } \\
\text { - Grupos de salud: desarrollo de grupos/redes de salud en } \\
\text { diferentes barrios. }\end{array}$ & \\
\hline \multirow{3}{*}{ Plus 55} & $\begin{array}{l}\text { Problemas: } \\
\text { - Recoger las voces de las personas mayores. } \\
\text { - Desconocimiento de planes y proyectos del resto. } \\
\text { - Llegar a sectores de la población a quienes no llegamos. } \\
\text { - Recursos económicos y físicos insuficientes. Optimizarlos. }\end{array}$ & \multirow{3}{*}{$\begin{array}{l}\text { - Información. } \\
\text { - Difusión. } \\
\text { - Conexión. } \\
\text { - Viabilización de campañas. } \\
\text { - Detección de perfiles diversos y derivación adecuada. } \\
\text { - Relaciones de confianza establecidas con las personas } \\
\text { mayores y asociaciones. }\end{array}$} \\
\hline & $\begin{array}{l}\text { Necesidades: } \\
\text { - Control información sobre/a la población objetivo. } \\
\text { - Conectar con perfiles diversos de >55. } \\
\text { - Creación de grupos/redes. } \\
\text { - Viabilizar propuestas de participación. } \\
\text { - Diversificar la información sobre Plus } 55 \text { desde diferentes } \\
\text { puntos y recursos físicos. }\end{array}$ & \\
\hline & $\begin{array}{l}\text { Planes: } \\
\text { - Llegar a perfiles diferentes de }>55 \text {. } \\
\text { - Prever futuras necesidades del colectivo. } \\
\text { - Mantener una línea estable del impulso de la participación } \\
\text { y del empoderamiento de las personas de }>55 \text {. } \\
\text { - Suministrar a las personas mayores herramientas para el } \\
\text { control de su vida. }\end{array}$ & \\
\hline \multirow{3}{*}{$\begin{array}{l}\text { Donostia } \\
\text { Lagunkoia }\end{array}$} & $\begin{array}{l}\text { Problemas: } \\
\text { - Llegar al territorio, conocer bien las necesidades, deseos, } \\
\text { expectativas de la gente (tanto de la ciudadanía, como de } \\
\text { quien está en el terreno). } \\
\text { - Incorporar/activar miradas más poliédricas. }\end{array}$ & \multirow{3}{*}{$\begin{array}{l}\text { - Información. } \\
\text { - Análisis. } \\
\text { - Acceso y relación con agentes muy diferentes. } \\
\text { - Conectar. } \\
\text { - Contribuir. } \\
\text { - Concienciar. } \\
\text { - Influir en quienes influyen. } \\
\text { - Identificar ventanas de oportunidad. }\end{array}$} \\
\hline & $\begin{array}{l}\text { Necesidades: } \\
\text { - Activar iniciativas y agentes alineados con objetivos y } \\
\text { formas de hacer de Donostia Lagunkoia. } \\
\text { - Cuestiones que impactan hoy, pero también poniendo en } \\
\text { la agenda de hoy las que van a impactar en el futuro. }\end{array}$ & \\
\hline & $\begin{array}{l}\text { Planes: } \\
\text { - Comunicar, impactar, inspirar, inspirarnos, activar/ } \\
\text { articular y proyectos. Hoy y con un horizonte de 15-20 } \\
\text { años. }\end{array}$ & \\
\hline \multirow{3}{*}{$\begin{array}{l}\text { Patronato de } \\
\text { Deportes del } \\
\text { Ayuntamiento } \\
\text { de Donostia- } \\
\text { San Sebastián }\end{array}$} & $\begin{array}{l}\text { Problemas: } \\
\text { - Hacemos nuestro el problema de la salud de la sociedad } \\
\text { (de parte). } \\
\text { - Buscamos un rendimiento social de los recursos públicos } \\
\text { que gestionamos. } \\
\text { - Personal justo. } \\
\text { - Prima lo urgente sobre lo importante. } \\
\text { - Subcontratación de la prestación directa. }\end{array}$ & \multirow{3}{*}{$\begin{array}{l}\text { - Cuadro de técnicos/as que atienden a las personas } \\
\text { (subcontratados). } \\
\text { - Red de equipamientos. } \\
\text { - Interés del Plan Municipal del Deporte. } \\
\text { - Acoger a otros agentes. }\end{array}$} \\
\hline & $\begin{array}{l}\text { Necesidades: } \\
\text { - Que el resto de colaboradores ayude a prescribir actividad } \\
\text { física. } \\
\text { - Mayor ocupación/utilización de recursos en horas valle. }\end{array}$ & \\
\hline & $\begin{array}{l}\text { Planes: } \\
\text { - Diseñar una red de prescripciones y acompañamientos. } \\
\text { - Adaptar los recursos a las personas (instalaciones). }\end{array}$ & \\
\hline
\end{tabular}




\begin{tabular}{|c|c|c|}
\hline \multirow{3}{*}{$\begin{array}{l}\text { Colegio } \\
\text { Oficial de } \\
\text { Farmacéuticos } \\
\text { de Gipuzkoa } \\
\text { (COFG) }\end{array}$} & $\begin{array}{l}\text { Problemas: } \\
\text { - Insuficiente integración de la farmacia en el equipo de } \\
\text { atención primaria. }\end{array}$ & \multirow{3}{*}{$\begin{array}{l}\text { - La red de farmacias comunitarias en Donostia-San } \\
\text { Sebastián (18). } \\
\text { - Accesibilidad de las farmacias. } \\
\text { - El COFG: apoyo y formación a las farmacias. } \\
\text { per un profesional sanitario cercano, conocido y accesible } \\
\text { para el paciente. Integrado en el equipo de salud. }\end{array}$} \\
\hline & $\begin{array}{l}\text { Necesidades: } \\
\text { - Mayor comunicación con el centro de salud. } \\
\text { - Programas en los que la farmacia, además de dar, recibe } \\
\text { un beneficio (no necesariamente económico). }\end{array}$ & \\
\hline & $\begin{array}{l}\text { Planes: } \\
\text { - Integrar más la farmacia en el equipo multidisciplinar } \\
\text { sociosanitario. }\end{array}$ & \\
\hline \multirow{3}{*}{ Anjel Irastorza } & $\begin{array}{l}\text { Problemas: } \\
\text { - Acceder a grupos de experimentación con proyectos de } \\
\text { innovación social. }\end{array}$ & \multirow{3}{*}{$\begin{array}{l}\text { - Tiempo: a delimitar. } \\
\text { - Expertise en trabajos con mejora de grupos y equipos. } \\
\text { Eficiencia y hábitos saludables. } \\
\text { - Expertise como instructor. Programa Paciente Activo, } \\
\text { conferencias, charlas motivacionales. } \\
\text { - Punto de vista/acción/representación independiente y/o } \\
\text { como agente de la sociedad civil. }\end{array}$} \\
\hline & $\begin{array}{l}\text { Necesidades: } \\
\text { - Integrarse en programas, proyectos y/o acciones } \\
\text { estructuradas, reconocibles y reconocidas. }\end{array}$ & \\
\hline & $\begin{array}{l}\text { Planes: } \\
\text { - Ser reconocido como agente de transformación del } \\
\text { desarrollo personal/organizacional saludable (visión } \\
\text { holística). }\end{array}$ & \\
\hline \multirow{3}{*}{$\begin{array}{l}\text { Acción Social } \\
\text { Ayuntamiento } \\
\text { de Donostia- } \\
\text { San Sebastián }\end{array}$} & $\begin{array}{l}\text { Problemas: } \\
\text { - Poca conciencia de enfermedad en determinados } \\
\text { colectivos. } \\
\text { - Poca adherencia a tratamientos de algunos colectivos. } \\
\text { - Detectar a personas con problemas de salud y sociales. } \\
\text { - No existencia de suficiente coordinación sociosanitaria en } \\
\text { intervenciones con ciertas personas. } \\
\text { - Participación de las personas en gestión de la salud. }\end{array}$ & \multirow{3}{*}{$\begin{array}{l}\text { - Técnicos/as conocedores de las necesidades sociales de } \\
\text { la comunidad. } \\
\text { - Técnicos/as motivadoras de sectores de la población para } \\
\text { participar en dinámicas comunitarias. } \\
\text { - Conocimiento y participación técnica en dinámicas } \\
\text { comunitarias. } \\
\text { - Mucha relación directa con la ciudadanía. }\end{array}$} \\
\hline & $\begin{array}{l}\text { Necesidades: } \\
\text { - Generar dinámicas saludables. } \\
\text { - Fomentar actividades con colectivos necesitados de } \\
\text { educación para la salud en diferentes etapas de su vida. } \\
\text { - Mejorar la coordinación entre los sistemas. } \\
\text { - Mejorar la detección. }\end{array}$ & \\
\hline & $\begin{array}{l}\text { Planes: } \\
\text { - Participar en las dinámicas comunitarias. } \\
\text { - Seguir fomentando la coordinación estructurada con } \\
\text { centros de salud (enfermería-médicos). }\end{array}$ & \\
\hline \multirow{3}{*}{$\begin{array}{l}\text { OSI } \\
\text { Donostialdea }\end{array}$} & $\begin{array}{l}\text { Problemas: } \\
\text { - Presión asistencial. } \\
\text { - Multiplicidad de otro tipo de programas y experiencias con } \\
\text { necesidad de resultados inmediatos. }\end{array}$ & \multirow{3}{*}{$\begin{array}{l}\text { - Conocimiento. } \\
\text { - Relación diaria directa con la comunidad. } \\
\text { - Concretar el respeto a las necesidades. }\end{array}$} \\
\hline & $\begin{array}{l}\text { Necesidades: } \\
\text { - Apoyo directivo en el reconocimiento concreto en cuanto a } \\
\text { la participación de actividades comunitarias. }\end{array}$ & \\
\hline & $\begin{array}{l}\text { Planes: } \\
\text { - Implementar el abordaje comunitario de la salud en la } \\
\text { práctica de los servicios. }\end{array}$ & \\
\hline \multirow{3}{*}{ Aptes } & $\begin{array}{l}\text { Problemas: } \\
\text { - Baja presencia en la sociedad. } \\
\text { - Bajo número de personas y entidades socias. } \\
\text { - Dificultad para vender nuestro "producto", valor, etc. } \\
\text { - Incorporar a gente joven en la asociación. }\end{array}$ & \multirow{3}{*}{$\begin{array}{l}\text { - Conocimiento de interlocutores en ámbitos diversos. } \\
\text { - Principios y herramientas de trabajo. } \\
\text { - Formación a directivos y gestores. } \\
\text { - Experiencia de quince años en proyectos de impacto } \\
\text { social. } \\
\text { - Independencia. } \\
\text { - Difusión en foros. }\end{array}$} \\
\hline & $\begin{array}{l}\text { Necesidades: } \\
\text { - Proyectos que aseguren nuestra sostenibilidad. } \\
\text { Euskaltrust como ejemplo de uno de ellos. } \\
\text { - Mayor red de prescriptores. }\end{array}$ & \\
\hline & $\begin{array}{l}\text { Planes: } \\
\text { - Ser referencia en Euskadi. } \\
\text { - Crear modelos innovadores de relación (formación-acción). }\end{array}$ & \\
\hline
\end{tabular}

Fuente: Elaboración propia. 


\section{Referencias bibliográficas}

ARGÜELLES, M.V. et al. (2016): Guía para trabajar en salud comunitaria en Asturias, Consejería de Sanidad del Principado de Asturias «https:// obsaludasturias.com/obsa/wp-content/ uploads/Guia_salud_comunitaria_2016.pdf〉.

BENITO, A. et al. (2016): Guía metodológica para el abordaje de la salud desde una perspectiva comunitaria, Vitoria-Gasteiz, Departamento de Salud del Gobierno Vasco, Osakidetza <http:// www.osakidetza.euskadi.eus/contenidos/ informacion/publicaciones_informes_estudio/ es_pub/adjuntos/guia-metodologia-esp.pdf〉.
DEPARTAMENTO DE SALUD DEL GOBIERNO VASCO (2013): Políticas de salud para Euskadi 20132020, Vitoria-Gasteiz, Servicio Central de Publicaciones del Gobierno Vasco <http://www. euskadi.eus/contenidos/plan_gubernamental/ xleg_plangub_13/es_plang_13/adjuntos/plan_ salud_2013_2020-web\%2012_03_2018.pdf).

HERNÁN, M. et al. (eds.) (2010): Formación en salutogénesis y activos para la salud, serie Monografías EASP, nำ 51, Escuela Andaluza de Salud Pública <https://www.easp.es/?wpdmact=process\&did $=$ Mi5ob3 $_{3}$ saW $_{5}$ r . 